\title{
Esforço percebido, estresse e inflamação do trato respiratório superior em atletas de elite de canoagem
}

CDD. 20.ed. 796.073

797.12

\author{
Alexandre MOREIRA* \\ Thiago Oliveira BORGES B******$^{* * * *}$ \\ Alvaro Acco KOSLOWSKI****** \\ Antonio Carlos SIMÕES \\ Valdir José BARBANTI* \\ *Escola de Educa- \\ ção Física e Esporte, \\ Universidade de São \\ Paulo. \\ ${ }^{* *}$ Centro de Educação \\ Física e Desportos, \\ Universidade Estadual \\ de Londrina. \\ ${ }^{* * *}$ Confederação Bra- \\ sileira de Canoagem.
}

\section{Resumo}

0 presente estudo investigou o comportamento do esforço percebido, percepção de fontes e sintomas de estresse e de ocorrência e incidência de sintomas associados a inflamações do trato respiratório superior (ITRS) em atletas de elite do sexo feminino de canoagem velocidade, durante sete semanas de preparação para um campeonato internacional. Para tanto foram utilizados os instrumentos de: medida de esforço percebido da sessão (EPS); o DALDA e o WURSS-21. A ANOVA de Friedman foi utilizada a fim de verificar a hipótese de igualdade entre as sete semanas de investigação para cada uma das variáveis. 0 nivel de significância foi estabelecido em 5\%. 0 teste de Wilcoxon foi utilizado como "post-hoc" com 0 ajuste de Bonferroni, quando necessário. Os resultados para EPS revelaram diferença significante entre as sete semanas de investigação. Foram localizadas diferenças entre as semanas recuperativas e as semanas de intensificação do treinamento. Diferenças significantes foram reveladas para os sintomas de estresse (Parte B do DALDA) sinalizando para a sensibilidade do instrumento para discriminar semanas de intensificação e semanas recuperativas de carga de treinamento. 0 WURSS-21 revelou diferenças significantes na ocorrência e incidência de ITRS entre a semana de maior volume de treinamento (semana 1) e as demais semanas investigadas, exceto com a semana 2. Os resultados permitem concluir que os instrumentos utilizados na presente investigação podem ser úteis para monitorar as alterações no esforço percebido da sessão, fadiga, percepção de sintomas de estresse e de ocorrências de ITRS.

UnITERMos: Canoagem; Inflamação do trato respiratório superior; Esforço percebido.

\section{Introdução}

O treinamento é uma atividade sistemática que visa proporcionar alterações morfológicas, metabólicas e funcionais que possibilitem o consequente incremento do rendimento. $\mathrm{O}$ treinamento esportivo deve ser entendido como um processo de longo prazo, orientado para o desenvolvimento mais elevado possível de características fisiológicas e psicológicas (BARBANTI, 2005). Neste processo, as açôes organizadas com o objetivo de proporcionar estímulos efetivos para o incremento do rendimento, podem ser entendidas como fontes de estresse. Em termos conceituais, o estresse fisiológico imposto ao organismo pelo treinamento pode ser considerado como uma combinação de influências positivas (condicionamento) e negativas (fadiga) no rendimento (Mujica, Busso, Lacoste, Barale, Geyssant \& Chatard, 1996).

$\mathrm{O}$ estresse no treinamento de alto rendimento também se revela como respostas psiconeurofisiológicas que designam o estado mental e sentimental / emocional dos atletas ao tomar conhecimento das barreiras competitivas que os obrigam a concentrar e canalizar energias excessivas. Doses moderadas de estresses podem produzir efeitos positivos, auxiliando nos treinamentos e nas açôes dos atletas para lidar de forma mais natural com os estados de ansiedades. Por outro lado, podem levá-los ao "burnout" que é produzido por respostas psiconeurofisiológicas de esgotamento por esforços frequentes e excessivos (ALIÑo, Alonso \& AlCOCER, 
1999). Este excesso relativo às diferentes fontes de estresse, também tem sido associado a alguns fenômenos habitualmente descritos e discutidos na literatura. Por exemplo, cargas intensas com insuficiente periodização dos intervalos de restabelecimento têm sido sugeridas como causa da síndrome do "overtraining" ou do "overreaching" (HOFFMAN \& KAMINSKY, 2000; KRAEMER, French, Paxton, Hakkinen, Volek, Sebastianelli, Putukian, Newton, Rubin, Gomez, Vescovi, Ratames, Fleck, Lynch \& Knuttgen, 2004). A intensificação do treinamento, deliberada ou não, pode levar o atleta a experimentar sensaçôes de fadiga aguda, alteração no padrão de sono, alimentação, problemas de concentração, alterações no estado de humor, bem como diminuição do rendimento (NEDERHOF, Zwerver, Brink, MeEusen \& LeMmink, 2008).

Estas alteraçōes estão amplamente relacionadas às modificações nos diversos sistemas do organismo por conta do incremento do estresse. O sistema imunológico, por exemplo, demonstra - se extremamente sensível ao estresse psicológico e fisiológico. Alguns estudos objetivaram investigar o efeito decorrente da intensificação do treinamento na função imune e respostas imunoendócrinas ao exercício de resistência (Robson-Ansley, Blannin \& GleEson, 2007; Verde, THOMAS \& SHEPHARD, 1992). Os achados revelaram que períodos sustentados de treinamento intensivo levam a uma depressão de vários aspectos da imunidade inata e adaptativa. No mesmo sentido, períodos intensivos de treinamento militar, que envolveram atividade física extenuante, deficiência de ingestão alimentar, privação de sono e estresse psicológico, também induziram alterações da função imunológica (CARINS \& BOOTH, 2002; Tiollier, Gomez-Marino, Burnat, Jouanin, BourRILHON, Filaire, Guezennec \& ChennaOUI, 2005).

Atletas de alto rendimento comumente intensificam o treinamento por alguns dias ou semanas em determinadas etapas da temporada com o objetivo de incrementar o rendimento (Halson \& Jeukendrup, 2004). Libicz, Mercier, Bigou, Le Gallais e Castex (2006) demonstraram evidências que competiçōes repetidas e seguidas de triátlon tem um efeito acumulativo negativo nos níveis basais da imunoglobulina salivar A (anticorpo predominante na superfície da mucosa), sugerindo que uma alta frequência de treinamento e competição contribuiria para a imunossupressão. Esta situação poderia levar a um estado de "overreaching" no qual o desempenho seria reduzido temporariamente. Este estado, por sua vez, aumentaria o risco do atleta em contrair infecções ou inflamaçōes. O possível incremento da probabilidade de se contrair infecções e/ou inflamações, levou, entre outras, à formação da "teoria da janela aberta" da imunossupressão (Koch, Wherry, Petersen, JOHNSON, STUART \& SEXTON, 2007).

$\mathrm{O}$ monitoramento regular das variáveis imunes e hormonais poderia prover ótimo diagnóstico para a avaliação do impacto do exercício agudo e crônico na saúde (GleEson, 2000; Pedersen \& BruUnsGaARD, 1995) e identificar atletas com maior risco para desenvolverem infecçōes ou inflamações. A principal limitação neste sentido é o alto custo para a realização deste processo. A utilização de questionários e testes de campo tem sido sugerida como ferramentas práticas e acessíveis com o objetivo de monitorar as respostas durante o processo de treinamento (CoutTs, Reaburn, Piva \& Murphy, 2007; Coutts, Slattery \& Wallace, 2007; Foster, 1998; Meeusen, Duclos, Gleeson, Rietjens, Steinacker \& URHAUSEN, 2006; NEDERHOF et al., 2008). Alterações no estado de humor e estresse crônico têm sido associadas com a supressão imunológica. A auto-avaliação do estresse no esporte tem demonstrado ser um meio confiável de monitorar as reaçôes dos atletas em diferentes etapas de treinamento (ROBSON-ANSLeY, BLANNIN \& GLEESON, 2007; RUSHALL, 1990).

Questionários e inventários vêm sendo frequentemente utilizados em estudos que buscam estabelecer relaçōes entre estresse de diferente natureza (psicológica ou fisiológica), respostas imunológicas, desempenho e saúde. Rushall (1990) propôs a utilização do Daily Analysis of Life Demands in Athletes (DALDA), utilizado em diversas investigações sobre o treinamento esportivo (AChten, Halson, Moseley, Rayson, Casey \& Jeukendrup, 2004; Halson, Bridge, Meeusen, Busschaert, GleEson, Jones \& JeUKendRup, 2002; Robson-Ansley, Blannin \& GleEson, 2007).

O WURSS-21 (Wisconsin Upper Respiratory Symptom Survey, Barret, Brown, Mundt, Safdar \& LEOTA, 2005), por outro lado, oferece a possibilidade de se extrair informaçóes relacionadas a sintomas de infecções ou inflamações no trato respiratório superior, decorrentes, possivelmente, da diminuição da função imune, resultante de diferentes fontes de estresse. No que diz respeito ao monitoramento da carga de treinamento, Foster (1998) e Foster, Florhaug, Franklin, Gottschall, Hrovatin, Parker, Doleshal e Dodge (2001) demonstraram a utilidade do método da medida do esforço percebido da sessão. Este método tem sido aceito como um indicador de carga de treinamento, fornecendo uma medida do produto do tempo da sessão de treinamento pela percepção do atleta em relação à carga efetivamente realizada e também como um instrumento complementar para o monitoramento e investigação da síndrome de "overtraining" ou 
"overreaching" (Foster, 1998; MEEUSEn et al., 2006; Pyne, Gleeson, McDonald, Clancy, Perry Junior \& FRICKER, 2000).

No Brasil, desconhecemos estudos que buscaram analisar o comportamento do esforço percebido da sessão de treinamento, da percepçáo de fontes e sintomas de estresse bem como da ocorrência e incidência de sintomas associados a inflamaçōes do trato respiratório superior durante um determinado período de treinamento em atletas de alto rendimento de canoagem. Assim, este estudo

\section{Método}

\section{Amostra}

Utilizou-se do método não-probabilístico casual para a seleção da amostra, composta por 11 atletas de alto nível do sexo feminino de canoagem velocidade (média e desvio padrão para idade $=21 \pm 4,7$ anos; massa corporal $=61,1 \pm 5,3 \mathrm{~kg}$ e estatura $=$ $163 \pm 1 \mathrm{~cm})$ em preparação para um campeonato internacional. Todas as atletas assinaram um termo de consentimento livre e esclarecido, aprovado pelo Comitê de Ética e Pesquisa da Faculdade de Ciências se propôs a investigar as possíveis alterações nas fontes e sintomas de estresse, ocorrência e incidência de sintomas de inflamação do trato respiratório superior e esforço percebido da carga de treinamento, em atletas de alto nível de canoagem velocidade, do sexo feminino, durante uma etapa de preparação para um campeonato internacional. A hipótese era que o comportamento da percepção das alterações dos sintomas de estresse e inflamação do trato respiratório superior estivesse no mesmo nível das diferentes magnitudes de esforço percebido.

QUADRO 1 - Característica da carga de treinamento pré-estabelecida para cada um dos sete microciclos (semanas) investigados.

\begin{tabular}{|l|c|c|c|c|c|c|c|}
\hline Período de preparação & Especial & \multicolumn{5}{|c|}{ Competitivo } \\
\hline Semana (microciclo) & 1 & 2 & 3 & 4 & 5 & 6 & 7 \\
\hline Característica da carga & Forte & Regenerativa & Forte & Forte & Regenerativa & Forte & Forte \\
\hline
\end{tabular}

\section{Esforço percebido da sessão}

O esforço percebido da sessão de treinamento foi quantificado através do cálculo do produto da duração da sessão, em minutos, e da medida da categoria de esforço percebido da sessão, utilizando-se do método proposto por FosTER (1998) registrado 30 minutos após o término de cada sessão de treinamento.

\section{Questionário de fontes e sintomas de estresse}

O Questionário DALDA foi preenchido ao final de cada semana de treinamento, conforme procedimento sugerido por ROBSON-ANSLEY, BLANNIN e GLEESOn (2007). O DALDA é dividido em duas partes, nomeadamente Parte A e Parte B, que representam as fontes de estresse e sintomas de estresse,
Médicas da Universidade Estadual de Campinas (248/2007).

\section{Delineamento experimental}

A duração da etapa de investigação do treinamento foi de sete semanas (QUADRO 1). Os registros de medida de esforço percebido da sessão foram realizados diariamente. Os questionários DALDA e WURSS-21 foram preenchidos ao final de cada uma das semanas investigadas.

\section{Questionário de sintomas de inflamações do trato respiratório superior}

OWURSS-21 com 21 questôes do tipo "survey" busca colher informações de dimensōes relacionadas à saúde, que são negativamente afetadas por resfriados comuns e inclui um item de severidade global ("quanto debilitado você se sente hoje ?"), 10 itens baseados em sintomas, nove itens funcionais relacionados à qualidade de vida e um item de modificação geral ("comparado com ontem, sinto que meu resfriado está...”). Todos os itens são baseados em uma escala "tipo-Likert" de severidade de 0 a 7. 


\section{Tratamento estatístico}

A ANOVA de Friedman foi utilizada a fim de verificar a hipótese de igualdade entre as sete semanas de investigação para cada uma das variáveis. O nível de significância foi estabelecido em 5\%. Quando necessário, o teste de Wilcoxon foi utilizado como "post-hoc" com o ajuste de Bonferroni. Foram analisadas separadamente as fontes de estresse (Parte A do DALDA) e os sintomas de estresse (Parte B do DALDA). Foram considerados os valores registrados para "pior do que o normal" em ambas as partes (A e B). Para o WURSS-21, os conceitos de "moderado" a "severo" (5 a 7) foram retidos para análise.

\section{Resultados}

A TABELA 1 apresenta a média e desvio padrão para cada uma das variáveis analisadas no estudo.

Os resultados do esforço percebido da sessão de treinamento revelaram diferenças significantes entre as sete semanas de investigação ( $\mathrm{X}^{2}$ [qui-quadrado] $=45,90$ ). As diferenças localizadas foram entre semana 1 e2, 1 e 5, 2 e3, 2e 4,2e6,2 e7,3e 5,4e 5, 5e6,5 e7,6e7 (FIGURA 1).

Não foram verificadas diferenças significantes para a parte A do questionário de DALDA, relativa às fontes de estresse, entretanto, diferenças significantes foram reveladas para a parte $\mathrm{B}$, relacionadas aos sintomas de estresse $\left(X^{2}=17,43\right.$; FIGURA 2). As diferenças localizadas foram para semana 1 e 2,1 e 3,1 e 5,1 e 7,4 e 5,5 e 6.

No que diz respeito ao WURSS-21, também se verificaram diferenças significantes entre as sete semanas $\left(X^{2}=20,66\right)$. As diferenças foram localizadas entre as semanas 1 e 3, 1 e 5, 1 e 6, 1 e 7 (FIGURA 3). Nas semanas 1 e 4 foram obtidas as maiores quantidades de sintomas com conceitos "moderado" a "severo".
* = Denota diferença em relação à semana 1 .

$¥=$ Diferente em relação à semana 5 . $€=$ Diferente em relação à semana $2 \mathrm{e} 5$.

$\beta=$ Diferente em relação à semana $6(P<0,05)$. DALDA = Daily Analysis of Life Demands in Athletes.

WURSS-21 = Wisconsin Upper Respiratory Symptom Survey. EPS = Medida do esfor ço percebido da sessão (valores semanais).
* Diferença significativa em relação a 2 e 5 ( $P$ $<0,05$ ).

$¥$ Diferença em relação a $6(P<0,05)$.
TABELA 1 - Média (X) e desvio padrão (DP) para os instrumentos DALDA, WURSS-21 e EPS, para cada uma das sete semanas de investigação.

\begin{tabular}{ccccccccc}
\hline \multirow{2}{*}{ Semanas } & \multicolumn{2}{c}{ DALDA - Fontes } & \multicolumn{2}{c}{ DALDA - Sintomas } & \multicolumn{2}{c}{ WURSS-21 } & \multicolumn{2}{c}{ EPS } \\
\cline { 2 - 8 } & $\mathrm{X}$ & $\mathrm{DP}$ & $\mathrm{X}$ & $\mathrm{DP}$ & $\mathrm{X}$ & $\mathrm{DP}$ & $\mathrm{X}$ & DP \\
\hline 1 & 1,45 & 1,04 & $6,45^{¥}$ & 2,16 & 4,55 & 3,27 & $655,49^{\epsilon}$ & 130,38 \\
2 & 0,73 & 1,01 & $2,73^{*}$ & 2,53 & 2,82 & 4,96 & 198,20 & 80,62 \\
3 & 0,91 & 1,22 & $3,18^{*}$ & 2,60 & $1,36^{*}$ & 2,34 & $653,03^{€}$ & 173,22 \\
4 & 0,73 & 1,27 & $4,09^{¥}$ & 3,24 & 5,55 & 6,99 & $573,38^{\epsilon}$ & 154,87 \\
5 & 1,18 & 1,47 & $1,82^{*}$ & 1,78 & $2,55^{*}$ & 5,96 & 243,19 & 71,64 \\
6 & 0,91 & 1,38 & $4,73^{* ¥}$ & 3,88 & $2,27^{*}$ & 3,13 & $712,05^{€}$ & 179,15 \\
7 & 0,91 & 1,22 & 3,55 & 2,11 & $2,36^{*}$ & 2,34 & $616,97^{€ \beta}$ & 158,74 \\
\hline
\end{tabular}

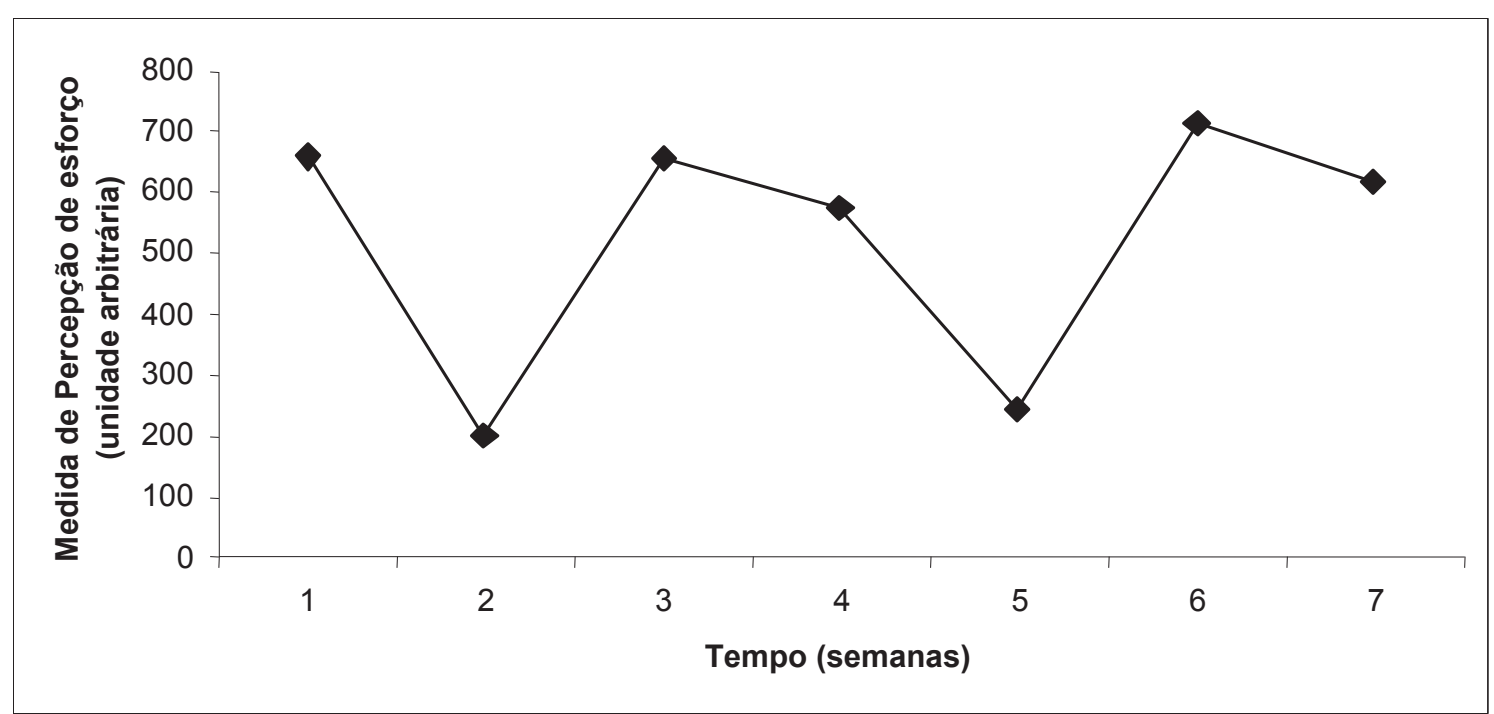

FIGURA 1 - Medida de percepção de esforço, determinada a partir dos procedimentos sugeridos por FosTER (1998). 


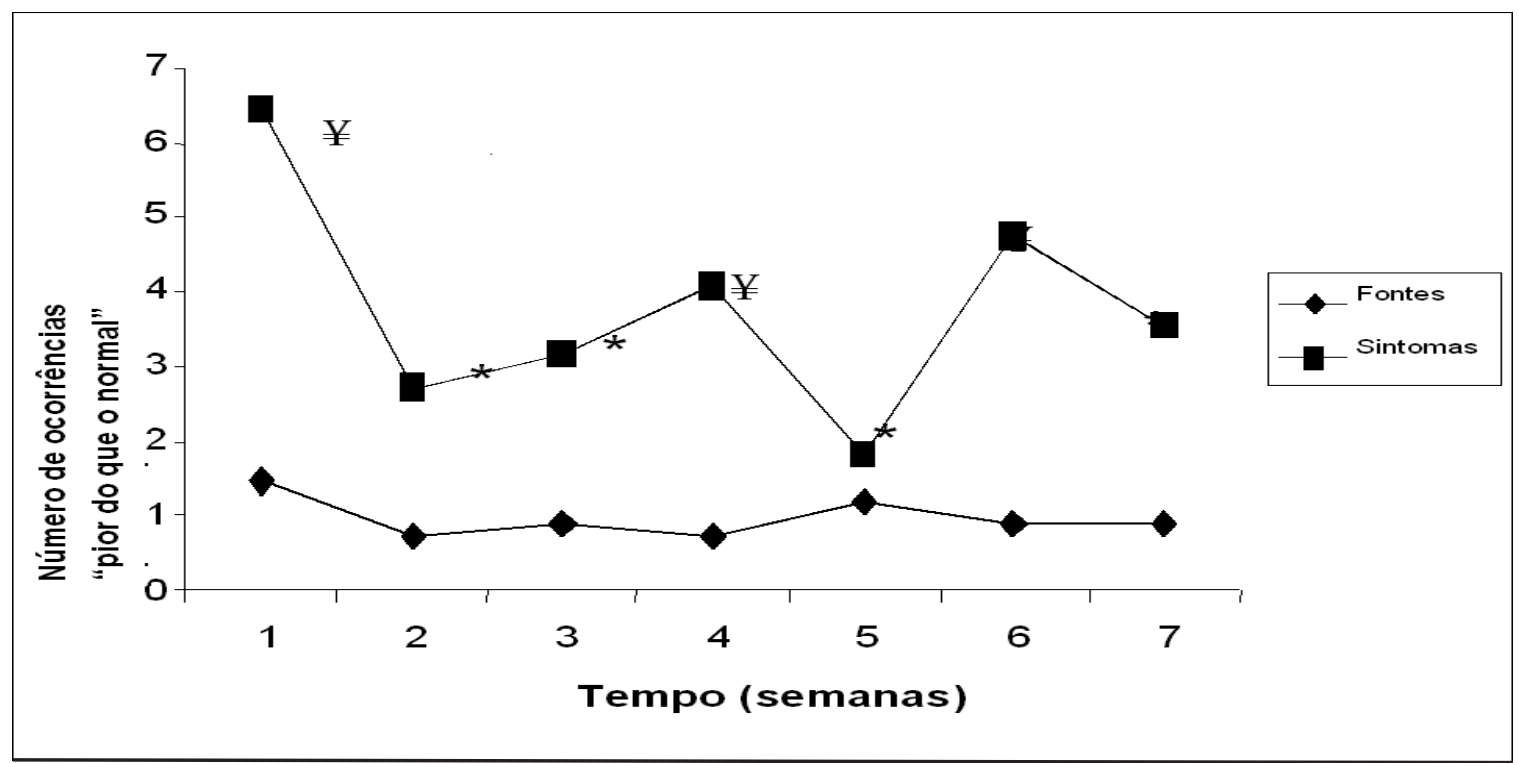

FIGURA 2 - Comportamento das ocorrências percebidas de fontes e sintomas de estresse (média).

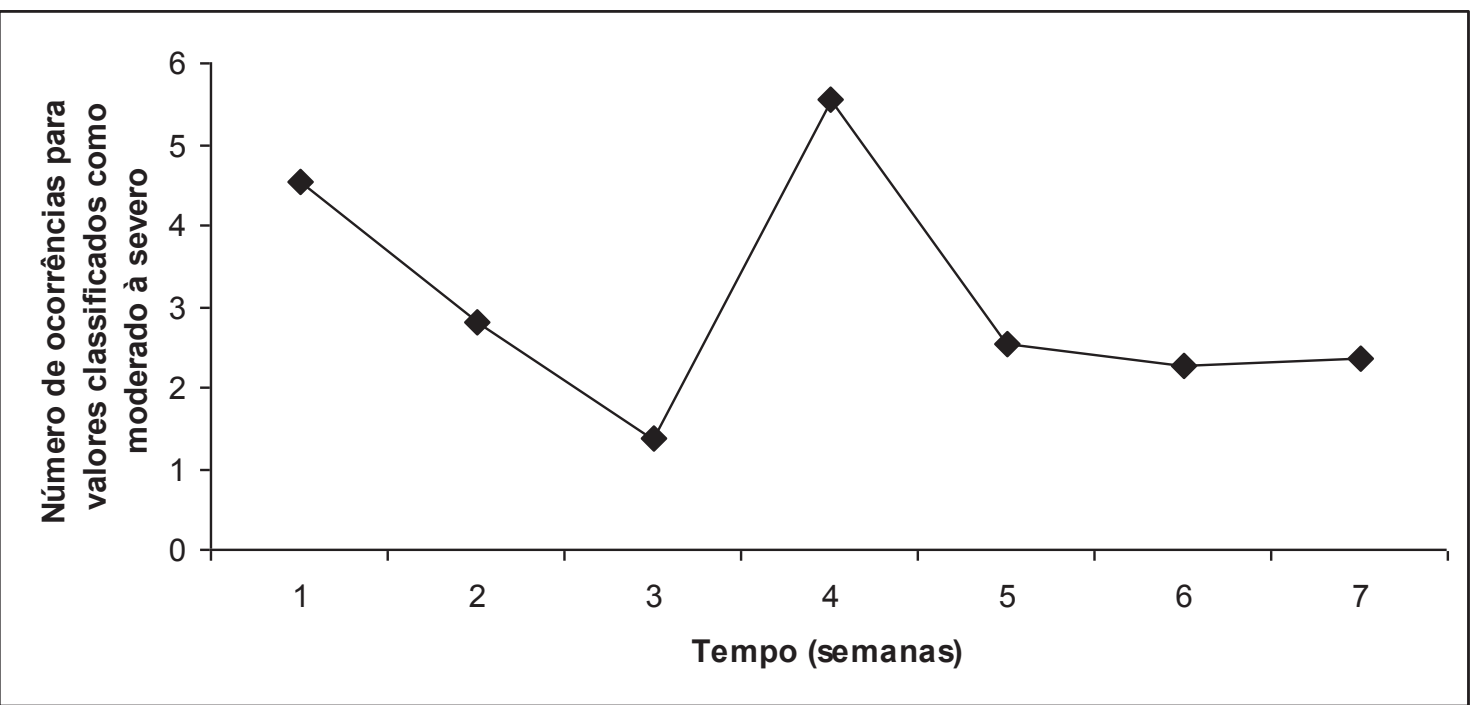

* Diferente em relação à semana $1(P<0,05)$.

FIGURA 3 - Comportamento dos valores médios “moderado" a "severo" obtidos do questionário WURSS-21.

\section{Discussão}

$\mathrm{Na}$ presente investigação, em concordância com outros estudos (ACHTEN et al., 2004; CoutTs et al., 2007; Halson et al., 2002; RobSON-ANSLEY, BLANNIN \& GLEESON, 2007), o questionário DALDA revelou-se sensível ao incremento e redução da carga de treinamento, no que se refere aos "sintomas de estresse" (Parte B). As fontes de estresse não se alteraram significativamente durante as sete semanas,
* Denotam diferença estatisticamente significante do conceito "pior do que o normal" em relação à semana $1(\mathrm{P}$ $<0,05$ );

$¥$ Denotam diferença estatisticamente significante em relação à semana $5(P<0,05)$

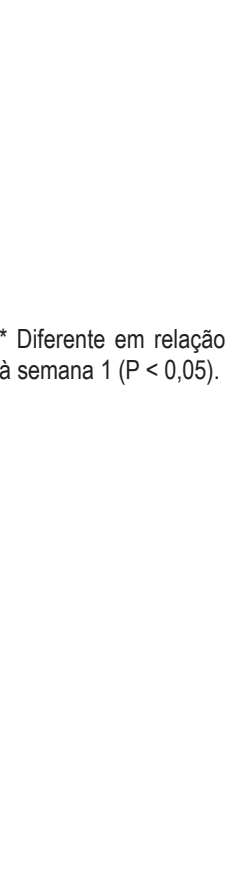


incremento significante dos "sintomas de estresse" uma semana antes das alterações nos marcadores que apontavam imunossupressão.

Também, do mesmo modo que no presente estudo, Coutts et al. (2007) reportaram a sensibilidade da Parte B do questionário DALDA no que concerne às alteraçôes da carga de treinamento. Os autores demonstraram incremento dos conceitos "pior do que o normal" da Parte B do DALDA quando intensificaram o treinamento, e uma diminuição nos momentos de recuperação.

A sensibilidade do DALDA é evidenciada quando se analisa sua dinâmica em relação às magnitudes de carga de treinamento propostas. Nas semanas regenerativas (semanas 2 e 5) revelou-se a menor quantidade de conceitos "pior do que o normal" na parte B do questionário. Evidenciou-se também diferenças significantes entre as semanas 2 e 5 , em relação a semana de maior volume de treinamento (semana 1 - último microciclo do período preparatório especial). Após a semana regenerativa (semana 2 ), foram realizados dois microciclos de intensificação do treinamento com evidente tendência de crescimento da quantidade de conceitos "pior do que o normal".

$\mathrm{O}$ indicador de carga de treinamento utilizado na presente investigação foi o método da medida do esforço percebido da sessão proposto por FosTER (1998); este método indica a percepção do atleta em relação à magnitude da carga de treinamento através dos valores relacionados ao produto da categoria da medida do esforço percebido da sessão de treinamento e o tempo de duração (em minutos). Os resultados encontrados demonstram uma dinâmica típica dos conceitos clássicos da periodização do treinamento, com alternância de semanas com cargas fortes e regenerativas. Os maiores valores de esforço percebido foram encontrados na primeira, terceira e quinta semanas do período analisado. Os valores elevados na primeira semana podem se justificar pelo fato deste momento corresponder ao final do denominado período preparatório especial e, consequentemente, apresentar um volume de cargas de treinamento maior em relação às semanas seguintes. Para as semanas 3 e 6 , o conteúdo de treinamento, apesar de diferenciado em relação à semana 1 (menor volume e maior intensidade), também objetivava incremento do estresse de treinamento.

Uma relação em forma de "J" entre o risco de se contrair infecções ou inflamaçôes do trato respiratório superior (ITRS) e a quantidade de exercício tem sido proposta, sugerindo que um nível moderado de exercício regular pode prover proteção contra episódios de ITRS, ao passo que níveis severos de exercício crônico parecem incrementar a incidência destes episódios acima dos níveis considerados normais (NIEMAN, 1997). O autor afirma ainda, que com um volume moderado de treinamento, o risco dos atletas contraírem ITRS é menor do que em indivíduos sedentários, entretanto, o risco é maior quando o volume de treinamento é substancial.

Um instrumento que possa auxiliar no monitoramento da ocorrência e incidência de inflamaçōes/ infecçôes do trato respiratório superior (ITRS) parece ser de grande utilidade para o processo de treinamento de atletas de alto rendimento. No presente estudo, os valores mais altos de conceitos "moderado" a "severo" no WURSS-21, aparecem na semana 1 e semana 4. É plausível admitir que a semana de maior volume de todo o período de investigação (semana 1) levou a um incremento na percepção dos sintomas de ITRS. No que diz respeito à semana 4 , parece que apesar de uma semana regenerativa (semana 2), o acúmulo de duas semanas de intensificação do treinamento (semanas 3 e 4) também induziu ao aumento na percepção dos sintomas.

PYNE et al. (2000) afirmam que incrementos vigorosos no treinamento, seja mediante ao aumento do volume, seja por conta da intensidade da carga de treinamento, ou ainda, de suas combinaçôes, são fatores adicionais de pressão para o sistema imunológico. Os autores sugerem que treinadores e profissionais que atuam com atletas de alto rendimento estejam alerta aos períodos de incremento do risco de ITRS e, que seja dada particular atenção à recuperação. Neste sentido, é razoável admitir a hipótese de que em caso de um novo microciclo de intensificação na semana 5 , ao invés do microciclo regenerativo efetivamente realizado, as atletas investigadas poderiam sinalizar com indicadores de excesso de treinamento, especialmente, uma elevação (ao invés da referida diminuição na semana 5) na percepção dos sintomas de ITRS, fato que poderia estar associado a imunossupressão decorrente do treinamento intenso.

Estudos longitudinais de curto prazo, que buscaram a intensificação do treinamento ou do incremento das fontes de estresses de diferentes naturezas (privação de sono, restrição alimentar, etc.) observaram o crescimento de sinais e sintomas de ITRS (CARins \& Booth, 2002; Tiollier et al., 2005). Ainda que não seja diagnosticado o patógeno e portanto, existindo limitação em assumir a ocorrência como infecção do trato respiratório 
superior, a diminuição da função da imunidade da mucosa após períodos de intensificação do treinamento poderia explicar em parte o incremento dos episódios associados a ITRS.

Um dos objetivos dos treinadores e outros profissionais que atuam com os atletas de alto rendimento é antecipar os fatores estressantes e através de uma apropriada avaliação, planificação, e monitoramento adequado, eliminar ou minimizar o impacto destes. Simples inflamaçōes do trato respiratório superior, apesar de serem importantes sinalizadores de alterações da função imune, requerem não mais do que alguma redução da carga de treinamento, no entanto, a continuidade de cargas intensas pode incrementar a severidade e a duração destes eventos. Fatores como exposição ao patógeno, lesões na mucosa, poluição do ar, entre outros, também podem influenciar o incremento do risco de se contrair inflamações ou infecções do trato respiratório superior; assim, é importante frisar que outras medidas devem ser tomadas no sentido de reverter a imunossupressão, pois somente a intensificação do treinamento, isoladamente, pode não explicar os aumentos dos episódios de ITRS.

\title{
Conclusão
}

Os achados do presente estudo permitem concluir que os instrumentos utilizados podem ser úteis para o monitoramento do processo de treinamento. $\mathrm{O}$ DALDA, no que diz respeito à Parte $\mathrm{B}$ (sintomas de estresse) se mostrou sensível às modificações da magnitude da carga de treinamento. O WURSS-21, por sua vez, sinalizou para o incremento do risco de inflamações do trato respiratório superior durante as semanas de intensificação do treinamento. A medida do esforço percebido da sessão evidenciou a mesma dinâmica do DALDA e do WURSS-21 ao longo da investigação. A hipótese que o comportamento da percepção das alterações dos sintomas de estresse e inflamação do trato respiratório superior estivesse no mesmo nível das diferentes magnitudes de esforço percebido foi corroborada.

\begin{abstract}
Perceived exertion, stress and upper respiratory tract illness in female elite canoeing flatwater

The aim of the present study was to identify the behavior of session rating of perceived exertion (session RPE), perception of sources and symptoms of stress and perception of occurrence and incidence of upper respiratory tract illness symptoms (URTI) in elite canoeing flatwater female athletes, during 7 weeks of preparation for an international championship. The methods used were the session RPE, DALDA and WURSS-21. The ANOVA of Friedman was applied on the dependent variables. The level of significance was established in 5\%. Wilcoxon test was used as post-hoc with the adjustment of Bonferroni when necessary. The session RPE showed significant differences between the taper weeks and the overload weeks (intensified training). Significant differences for the Part B of the DALDA (symptoms of stress) have been demonstrated. To URTI symptoms, significant differences were between the week of greater volume of training (week 1) and the remaining of investigated weeks, except with week 2 . The results allow concluding that the instruments used in the present investigation may be useful for assessing changes in perceptual training load, fatigue, stress reaction symptoms and occurrence of perceived URTI symptoms.
\end{abstract}

UnITERMS: Canoeing; Upper respiratory tract illness; Rate of perceived exertion. 


\section{Referências}

ACHTEN, J.; HALSON, S.L.; MOSELEY, L.; RAYSON, M.P.; CASEY, A.; JEUKENDRUP, A.E. Higher dietary carbohydrate content during intensified running training results in better maintenance of performance and mood state. Journal of Applied Physiology, Washington, v.96, n.4, p.1331-40, 2004.

ALIÑO, J.J.L.I.; ALONSO, T.O.; ALCOCER, M.I.LI. Leciones de psicologia médica. Barcelona: Masson, 1999.

BARBANTI, V. Formação de esportistas. São Paulo: Manole, 2005.

BARRET, B.; BROWN, R.; MUNDT, M.; SAFDAR, N.; LEOTA, DYE. The Wisconsin upper respiratory symptom survey is responsive, reliable, and valid. Journal of Clinical Epidemiology, New York, v.58, n.6, p.609-17, 2005.

CARINS, J.; BOOTH, C. Salivary immunoglobulin-A as a marker of stress during strenuous physical training. Aviation, Space, and Environmental Medicine, Washington, v.73, n.12, p.1203-7, 2002.

COUTTS, A.; REABURN, P.; PIVA, T.J.; MURPHY, A. Changes in selected biochemical, muscular strength, power, and endurance measures during deliberate overreaching and tapering in rugby league players. International Journal of Sports Medicine, Stuttgart, v.28, n.2, p.116-24, 2007

COUTTS, A.J.; SLATTERY, K.M.; WALLACE, L.K. Practical tests for monitoring performance, fatigue and recovery in triathletes. Journal of Science and Medicine in Sport, Belconnen, v.10, n.6, p.372-81, 2007.

FOSTER, C. Monitoring training in athletes with reference to overtraining syndrome. Medicine and Science in Sports and Exercise, Hagerstown, v.30, n.7, p.1164-8, 1998.

FOSTER, C.; FLORHAUG, J.A.; FRANKLIN, J.; GOTTSCHALL, L.; HROVATIN, L.A.; PARKER, S.; DOLESHAL, P.; DODGE, C. A new approach to monitoring exercise training. Journal of Strength and Conditioning Research, Champaign, v.15, n.1, p.109-15, 2001.

GLEESON, M. Mucosal immunity and respiratory illness in elite athletes. International Journal of Sports Medicine, Stuttgart, v.21, p.S33-43, 2000. Supplement 1.

HALSON, S.L.; BRIDGE, M.W.; MEEUSEN, R.; BUSSCHAERT, B.; GLEESON, M.; JONES, D.A.; JEUKENDRUP, A.E. Time course of performance changes and fatigue markers during intensified training in trained cyclists. Journal of Applied Physiology, Washington, v.93, n.3, p.947-56, 2002.

HALSON, S.L.; JEUKENDRUP, A.E. Does overtraining exist?: An analysis of overreaching and overtraining research. Sports Medicine, Auckland, v.34, n.14, p.967-81, 2004.

HOFFMAN, J.R.; KAMINSKY, M. Use of performance testing for monitoring overtraining in elite youth basketball players. Strength and Conditioning Journal, Champaign, v.22, n.6, p.54-62, 2000.

KRAEMER, W.J.; FRENCH, D.N.; PAXTON, N.J.; HAKKINEN, K.; VOLEK, J.S.; SEBASTIANELLI, W.J.; PUTUKIAN, M.; NEWTON, R.U.; RUBIN, M.R.; GÓMEZ, A.L.; VESCOVI, J.D.; RATAMES, N.A.; FLECK, S.J.; LYNCH, J.M.; KNUTTGEN, H.G. Changes in exercise performance and hormonal concentrations over a big ten-soccer season in starters and nonstarters. Journal of Strength and Conditioning Research, Champaign, v.18, n.1, p.121-8, 2004 KOCH, A.J.; WHERRY, A.D.; PETERSEN, M.C.; JOHNSON, J.C.; STUART, M.K.; SEXTON, W.L Salivary immunoglobulin A response to collegiate rugby game. Journal of Strength and Conditioning Research, Champaign, v.21, n.1, p.86-90, 2007. LIBICZ, S.; MERCIER, B.; BIGOU, N.; LE GALLAIS, D.; CASTEX, F. Salivary IgA response of triathletes participating in the French iron tour. International Journal of Sports Medicine, Stuttgart, v.27, n.5, p.389-4, 2006.

MEEUSEN, R.; DUCLOS, M.; GLEESON, M.; RIETJENS, G.; STEINACKER, J.; URHAUSEN, A. Prevention, diagnosis and treatment of overtraining syndrome. European Journal of Sports Science, London, v.6, n.1, p.1-14, 2006 MUJICA I, BUSSO T, LACOSTE L, BARALE F, GEYSSANT A, CHATARD JC. Modeled response to training and taper in competitive swimmers. Medicine and Science in Sports and Exercise, Hagerstown, v.28, n.2, p.251-8, 1996. NEDERHOF, E.; ZWERVER, J.; BRINK, M.; MEEUSEN, R.; LEMMINK, K. Different diagnostic tools in nonfunctional overreaching. International Journal of Sports Medicine, Stuttgart, v.29, n.7, p.590-7, 2008.

NIEMAN, D.C. Immune response to heavy exertion. Journal of Applied Physiology, Washington, v.2, p.1385-94, 1997. PEDERSEN, B.K.; BRUUNSGAARD, H. How physical exercise influences the establishment of infections. Sports Medicine, Auckland, v.19, n.6, p.393-400, 1995

PYNE, D.B.; GLEESON, M.; McDONALD, W.A.; CLANCY, R.L.; PERRY Jr, C.; FRICKER, P.A. Training strategies to maintain immunocompetence in athletes. International Journal of Sports Medicine, Stuttgart, v.21, p.S51-60, 2000. Supplement 1. ROBSON-ANSLEY, P.; BLANNIN, A.; GLEESON, M. Elevated plasma interleukin-6 levels in trained male triathletes following an acute period of intense interval training. European Journal of Applied Physiology, Berlin, v.99, n.4, p.353-60, 2007. 
RUSHAL, B.S. A tool for measuring stress tolerance in elite athletes. Journal of Applied Sports Psychology, Philadelphia, v.2, p.51-66, 1990.

TIOLLIER, E.; GOMEZ-MARINO, D.; BURNAT, P.; JOUANIN, J.C.; BOURRILHON, C.; FILAIRE, E.; GUEZENNEC, C.Y.; CHENNAOUI, M. Intense training: mucosal immunity and incidence of respiratory infections. European Journal of Applied Physiology, Berlin, v.93, n.4, p.421-8, 2005.

VERDE, T.; THOMAS, S.; SHEPHARD, R.J. Potential markers of heavy training in highly trained endurance runners.

British Journal of Sports Medicine, Loughborough, v.26, n.3, p.167-75, 1992.

\begin{tabular}{r|r} 
ENDEREço & \\
Alexandre Moreira & \\
Departamento de Esporte & \\
Escola de Educação Física e Esporte - USP & Recebido para publicação: 07/08/2008 \\
Av. Prof. Mello Moraes, 65 & Aceito: 04/08/2009 \\
085508-030 - São Paulo - SP - BRASIL & \\
e-mail: alemoreira@usp.br &
\end{tabular}

